\title{
Féeries
}

Études sur le conte merveilleux, XVII $-\mathrm{XIXe}$ siècle

$8 \mid 2011$

Le merveilleux français à travers les siècles, les langues, les continents

\section{Marvels \& Tales, Journal of Fairy-Tale Studies}

Détroit, Wayne State University Press, vol. 22 (2008), 23 (2009), 24 (2010)

Jean-François Perrin

\section{(2) OpenEdition}

\section{Journals}

Electronic version

URL: http://journals.openedition.org/feeries/783

DOI: $10.4000 /$ feeries.783

ISSN: 1957-7753

Publisher

UGA Éditions/Université Grenoble Alpes

Printed version

Date of publication: 15 October 2011

Number of pages: 195-201

ISBN: 978-2-84310-211-0

ISSN: $1766-2842$

Electronic reference

Jean-François Perrin, « Marvels \& Tales, Journal of Fairy-Tale Studies », Féeries [Online], 8 | 2011,

Online since 15 April 2013, connection on 23 September 2020. URL : http://journals.openedition.org/ feeries/783 ; DOI : https://doi.org/10.4000/feeries.783

\section{(c) Féeries}




\section{COMPTE RENDU CRITIQUe}

Marvels \& Tales, Journal of Fairy-Tale Studies, Détroit, Wayne State University Press, vol. 22 (2008), 23 (2009), 24 (2010).

Vol. 22 (2008), $\mathbf{n}^{\circ}$ I. Ce numéro spécial de Marvels \& Tales est consacré aux contes érotiques. Observant, dans sa dense présentation, qu'en dépit de leur relation centrale avec le désir, les contes de fées autant que les contes populaires ne sont pas assez approchés du point de vue érotique, Cristina Bacchilega ${ }^{\mathrm{I}}$ rattache cela à l'infantilisation moderne de ces genres et à la pruderie prudente des spécialistes du conte populaire - certains spécialistes des Mille et Une Nuits mis à part. Du côté de la critique littéraire moderne, en revanche, une approche informée par la psychanalyse dégage les enjeux sexuels latents de toute cette matière narrative, bien audelà des contes érotiques proprement dits. Les articles de ce numéro spécial étudient donc comment certains écrivains contemporains ont exploité la puissance libidinale des contes de fées. Renvoyant aux travaux pionniers d'Angela Carter $^{2}$, très inscrits dans la critique féministe des années 1970I980, C. Bacchilega rappelle ce que leur doit la problématique qu'elle défend ici : comment le désir sexuel est-il représenté dans le conte populaire ou le conte de fées? De qui la vie, la subjectivité et le plaisir sont-ils le centre du récit? Quelle est l'économie du sexe et du pouvoir dans le texte? Est-ce la violence qui l'éroticise? Le conte thématise-t-il le désir comme un ou comme multiple? Comment lisons-nous le récit depuis un autre espace désirant?

I. Voir son Postmodern Fairy Tales: Gender and Narratives Strategies, Philadelphie, University of Pennsylvania Press, 1997.

2. The Sadeian Woman and the Ideology of Pornography (New York, Pantheon, 1979), The Bloody Chamber and other Stories (Harmondsworth, Penguin, 1979), éd. The Virago Book of Fairy Tales (Londres, Virago Press, 1990). 
Comment la chair se fait-elle verbe? Telle est donc, en un mot, la question traitée dans la plupart des articles de ce numéro spécial. Quatre études abordent la sexualisation explicite de contes de fées dans un certain nombre d'œuvres contemporaines. Adam Zolkover analyse Fables de Billy Willingham, une sorte de roman-BD de 2002, où des personnages comme Blanche-Neige mènent une double vie plutôt agitée entre monde merveilleux et New York. Linda J. Lee consacre son étude aux récits " paranormaux" où des éléments de merveilleux sont mis au service d'intrigues érotiques hétérosexuelles souvent basées sur La Belle et la Bête. Sarah Lash étudie la trilogie d'Anne Rice, Sleeping Beauty, exemple bien connu de traitement érotique du conte. Jeana Jorgensen confronte les travaux d'A. Carter à d'autres recueils de contes de fées érotisés qui «ne problématisent pas le désir qu'ils représentent». Les questions ici posées ne sont pas simplement métanarratives (comment la figure d'un personnage de conte de fées est-elle adaptée à ces contes érotiques?), mais métafictionnelles : est-ce que les différents genres qui jouent sur l'attrait du conte énoncent quelque chose sur ce genre lui-même, et si tel est le cas, comment se négocie alors le rapport entre celui-ci et l'idéologie du "genre»?

Trois autres articles traitent de l'intertextualité dans les contes de fées érotiques. Kimberly J. Lau observe le jeu de l'instabilité dans la WolfTrilogy d'A. Carter. Shuli Barzilai étudie les machineries du désir dans Bluebeard's Keys d'Anne Thackeray Ritchie (XIX ${ }^{\mathrm{e}}$ siècle) et dans The Bloody Chamber d'A. Carter. Lisa G. Probst étudie trois ouvres de Marina Warner, qui travaillent la violence sexuelle dans les contes et les mythes, rencontrant ici l'intérêt de A. Carter pour une sexualité féminine aussi expansive que discrètement illicite. La méditation sur ce qu'il en est du rapport au pouvoir du désir féminin, ainsi que le débat avec la psychanalyse se trouvent en effet au centre de ces analyses, du moins si l'on accorde à A. Carter que "le conte de fées est dédié au principe de plaisir». Pauline Greenhill, enfin, révèle la transsexualité à l'œuvre dans le conte $n^{\circ} 46$ des frères Grimm (L'Oiseau de Fitcher) comme stratégie de survie.

Vol. 22 (2008), $\mathbf{n}^{\circ}$ 2. Au sommaire de ce numéro : un article de Tison Pugh sur l'utopie queer et l'érotisme antisocial dans Le Magicien d'Oz (I900) de Lyman Frank Baum. Une étude d'Ulrich Marzolph sur le conte de L'homme pieux et sa chaste épouse dans les Mille et Une Nuits, ses rapports avec Crescentia (conte type AT 712 chez Aarne-Thomson) et leur ensourcement arabe, persan et juif. Un article de Carolyn Fay sur l'intrigue de La Belle au bois dormant de Perrault, montrant le lien profond entre le sommeil de l'hérö̈ne et la tentative de meurtre par l'ogresse : la 
femme qui se soustrait au monde, soit par le sommeil soit par la voracité, serait un danger pour la société et pour le récit. Une étude, enfin, de Cristin Bildoshi sur quatre variantes russes du conte type ATU ${ }_{425} \mathrm{C}$, Beauty and the Beast.

Dans la rubrique "Texts \& Translations", on découvre avec intérêt une traduction de La Princessejalouse de Jean-Pierre Camus par AnneE. Duggan. Cette nouvelle peut se lire en français dans L'Amphithéâtre sanglant du même auteur (Paris, I630). On sait quelle contribution la plume prolifique de l'évêque de Belley apporte, entre autres, au genre de l'histoire tragique en vogue au XVII ${ }^{\mathrm{e}}$ siècle (toujours pour la bonne cause, cela va de soi chez ce fervent contre-réformateur disciple de François de Sales!). La thèse soutenue par la traductrice est que cette nouvelle constitue une source seconde de La Belle au bois dormant (la première étant Soleil, Lune et Talie de Basile). Perrault, qui avait lu Camus, aurait surtout emprunté à cette nouvelle la matière de la «seconde» intrigue impliquant la mère ogresse du prince : comme Camus en effet, il introduit une femme monstrueuse génératrice de désastre au moment précis où le prince étant appelé à la guerre, l'autorité masculine se trouve provisoirement suspendue dans son royaume.

Vol. 23 (2009), $\mathbf{n}^{\circ}$ I. Ce numéro est consacré à la traduction du conte de fées. Il présente des articles sur l'histoire de cette pratique, des traductions de contes européens, japonais et hawaïens, des traductions d'essais importants sur la traduction.

Bryan Kuwada étudie une traduction hawaïenne de Barbe bleue en I862, dans le journal Ka Nupepa Kuokoa. Martine Hennard Dutheil et Ute Heidmann analysent la traduction du même conte par Angela Carter; elles montrent qu'il s'agit au fond d'une réécriture dont l'enjeu s'éclaire par une comparaison avec The Bloody Chamber du même auteur.

Paul Buczkowski présente une passionnante analyse de la traduction par James Robinson Planché de l'intégrale des contes de Madame d'Aulnoy (récits-cadres compris) : Fairy Tales of the Countess d'Aulnoy (I855) ${ }^{3}$. Cet auteur, qui avait composé des fantaisies musicales basées sur les contes de fées, avait adapté pour la scène les scénarios de quatorze contes de Madame d'Aulnoy. Il était par ailleurs un spécialiste reconnu de l'Ancien Régime pour ce qui touchait aux mœurs, coutumes vestimentaires et alimentaires, arts et littérature, etc. Trouvant très insuffisantes les nombreuses

3. J. R. Planché (trad.), Fairy Tales of the Countess d'Aulnoy, Londres, G. Routledge \& Sons, I855; rééd. D’Aulnoy's Fairy Tales, Philadelphie, David McKay Compagny, I923. 
traductions de son auteur fétiche circulant en Angleterre depuis le début du XVIII ${ }^{e}$ siècle, il entreprit d'en donner une traduction nouvelle aussi précise et scrupuleuse que possible, respectant les encadrements romanesques des recueils, et cherchant les meilleures transpositions possibles de la langue baroque de Madame d'Aulnoy (y compris ses vers), notamment son phrasé complexe, sa fantaisie verbale et son esprit. Il munit également sa traduction d'un appareil de notes très fouillé éclairant des points d'histoire, des allusions contemporaines, des choix onomastiques, des idiomatismes, etc. Si l'entreprise est dans l'ensemble tout à fait réussie, anticipant d'ailleurs sur toute une série de préoccupations qui furent par la suite celles de Philippe Hourcade dans sa propre édition critique de Madame d'Aulnoy $^{4}$, cet article montre aussi comment elle reflète les idiosyncrasies de J. R. Planché, sa prudence critique et les limites que lui impose le manque de compétence des milieux académiques de son temps pour une discussion réelle de ses recherches.

Dans la rubrique "Texts \& Translations", on lira Umiumi Uliuli, traduction de la traduction hawaïenne de Barbe bleue évoquée plus haut; celle d'un conte de Gianni Rodari, le meilleur auteur de littérature de jeunesse italien selon Jack Zipes: The War of the Bells, tiré de ses Favole al Telefono ("Contes dits au téléphone»), 1962; celle d'un conte de la romancière japonaise Awa Naoko: Hanamame no nieru made ("Jusqu'à ce que les haricots soient cuits»), conte-titre d'un recueil de 1993; celle de deux contes occitans traditionnels : Le Roi des poissons et La Sorcière publiés en 1899 par Louis Lambert; celle de La Muñequita ("La Petite Poupée») de Juan Valera y Alcalá-Galiano, un des meilleurs écrivains espagnols du $\mathrm{XIX}^{\mathrm{e}}$ siècle : il s'agit d'une relecture ironique du conte type La Poupée qui mord, bien illustré notamment par Basile; celle de El rey en busca de novia de Antonio de Trueba, autre écrivain espagnol du XIX ${ }^{e}$ siècle qui collectait des contes populaires mais les trouvait souvent chez les frères Grimm, comme celui-ci, proche de leur Le Fuseau, la navette et l'aiguille; celle, enfin, de Las tres perlas ("Les trois Perles») d'un jésuite espagnol de la fin du XIX ${ }^{e}$ siècle, Luis Coloma S. J. proche des frères Grimm par l'esprit dans ses contes pour les enfants.

On redécouvrira enfin avec intérêt, et en anglais, la belle étude de Théophile Gautier sur les contes d'Hoffmann parue dans La Chronique de Paris (I836) et le "Dialogue sur les contes de fées» inclus par Anatole France dans Le Livre de mon ami (I885).

4. Madame d'Aulnoy, Contes I, II, Édition du Tricentenaire, Paris, STFM, I997. 
Vol. 23 (2009), $\mathbf{n}^{\circ}$ 2. Ce numéro offre d'abord un article de Kathleen Ragan consacré au "genre» des protagonistes de contes populaires publiés, articulé à celui des éditeurs, des collectionneurs et des écrivains. La représentation différentielle des contes populaires féminins y est statistiquement quantifiée à partir d'un corpus d'un millier de contes : selon l'auteur, la prise en compte du "genre" semble désormais indispensable, on le devine! à toute analyse sérieuse de cette matière. On lit ensuite un passionnant (et amusant) article de Maria Nikolajeva sur la représentation du chat dans la littérature et le folklore depuis l'Égypte ancienne jusqu'à nos jours. Sans prétendre à l'exhaustivité, l'auteur s'attache à relever les motifs et les rôles, les valences et les polarités, interrogeant constamment le trouble et constant rapport mythico-littéraire que l'homme entretient avec cet animal fort énigmatique.

On découvrira également une étude de Victoria Ivleva sur la fonction des textiles et des accessoires vestimentaires dans le recueil d'Afanassiev; un article de Gina M. Miele sur l'inscription vériste des détails du quotidien dans les contes de l'écrivain sicilien Luigi Capuana (cinq des contes de cet auteur de la fin du XIX ${ }^{\mathrm{e}}$ siècle sont traduits par Jack Zipes dans la rubrique "Texts \& Translations») ; une analyse de The Grimm's Sisters (I98I) de Liz Lochhead par Tudor Balinisteanu, et enfin une étude de Jacques Barchilon sur les contes types repérables dans l'œuvre de Madame d'Aulnoy.

Outre la traduction de L. Capuana déjà mentionnée, la rubrique «Texts \& Translations» offre également une traduction de deux poèmes du grand écrivain cubain contemporain Eliseo Diego, le premier reprenant Le Petit Chaperon rouge du point de vue du loup, le second évoquant Ma mère l'oie en narratrice de toute Origine.

Vol. 24 (20I0), $\mathbf{n}^{\circ}$ I. Ce numéro spécial de Marvels \& Tales est consacré au conte de fées après Angela Carter. Il est issu d'un colloque tenu en avril 2009 à l'université d'East Anglia (UK), à l'occasion du trentième anniversaire de la publication de The Bloody Chamber, ce fameux recueil qui a profondément transformé l'approche générale du genre.

Selon les éditeurs, Stephen Benson et Andrew Teverson, l'un des thèmes les plus insistants de la postérité critique d'A. Carter est la relation entre le conte de fées et l'idéologie nationaliste ou colonialiste. Donald Haase appelle ainsi à "décoloniser» les études sur le conte de fées; Sara Hine étudie comment la pratique anthologique des Fairy Books d'Andrew Lang (I889-I9Io) corrobore le discours du XIX ${ }^{\mathrm{e}}$ siècle sur le colonialisme et l'empire; Vassilena Parashkevova aborde, quant à elle, la signification culturelle du conte, en étudiant l'engagement subversif de Githa Hariharan à 
l'égard de l'approche de la voix des femmes dans les Mille et Une Nuits, avec son roman When Dream Travel (1999).

Un autre terrain consistant de la recherche sur le conte concerne ses rapports avec le cinéma. C'est ce qu'aborde Susan Cahill qui travaille sur la représentation de la vieille femme dans Les Frères Grimm de Terry Gilliam (2005) et Stardust de Matthew Vaughn (2007), chacun en référence à Blanche-Neige. Sharon McCann analyse pour sa part le film de Neil Jordan, The Company of Wolves, en montrant que le souci principal du cinéaste est moins la psyché féminine comme l'a généralement prétendu la critique, que l'histoire irlandaise et ses "tropes» littéraires dont ses autres films sont également hantés.

Touchant la prise en compte des écrivains qui, comme A. Carter, utilisent le conte comme tremplin narratif, de nouvelles perspectives apparaissent sur le rôle grandissant du conte dans les fictions récentes. La lecture du travail de Githa Hariharan par V. Parashkevova s'inscrit dans ce champ, comme aussi l'analyse par Jennifer Orme (dans une perspective queer) du recueil d'Emma Donoghue: Kissing the Witch (1997).

Enfin, un intérêt croissant se manifeste, dans les études sur A. Carter, pour la question de la traduction. C'est la matière de l'article de Martine Hennard Dutheil de la Rochère, centré sur la traduction de La Belle au bois dormant de Perrault par A. Carter, où elle montre que celle-ci subvertit moins les contes de Perrault comme on le pense généralement, qu'elle ne les annexe au féminisme parce qu'elle y reconnaît une constante volonté de familiariser les enfants avec la politique de l'expérience. À bien des égards, le thème de la traduction et de la traductibilité des contes de fées élargit la question de leur signification culturelle et nationale, abordée de façons variées par D. Haase, J. C. Hines, V. Parashkevova et S. McCann.

Ce qui, en retour, signale ce qui fut probablement le principal thème émergent du colloque : le rapport entre conte de fées et identité culturelle, et la capacité des contes à franchir ou non les frontières nationales. Ces problèmes de propriété, de transfert culturel, de spécificités culturelles et d'appropriation sont revenus de façon récurrente, et sont appelés à nourrir bien des interrogations à venir chez les spécialistes du champ.

Vol. 24 (20I0), $\mathbf{n}^{\circ}$ 2. Ce numéro offre six études. Le film Pan's Labyrinth de Guillermo del Toro (2006) y fait l'objet de deux articles : Jennifer Orme y étudie la relation entre le désir narratif et la désobéissance; cette relation s'articule dans la résistance de la jeune héroöne à la domination politique et familiale qu'elle affronte, autant qu'au trouble que le film lui-même introduit aux frontières génériques et dans les modalités discursives, en 
démultipliant versions et scénarios. Kristine Kotecki en travaille pour sa part les sources littéraires et contiques, pour ce que leur réappropriation cinématographique procure d'efficacité en termes de critique sociale.

Andrew Teverson étudie l'utilisation intertextuelle des contes Jack et le haricot magique et Whittington et son chat dans le roman de Samuel Selvons : Turn again Tiger (1958); cette réappropriation est interprétée dans une perspective post-colonialiste. Christy Williams étudie la méchante marâtre comme héroïne de conte, à travers le traitement peu classique qu'en propose le roman de Robert Coover : Stepmother (2004), ici abordé comme un "roman-conte de fées post-moderne». Laurence TalairachVielmas examine trois contes de fees victoriens : Beauty and the Beast de Anne Thackeray Ritchie (I867), The Ogre Courting de Juliana Horatia Ewing (187I), The Brown Bull of Norrowa de Mary Louisa Molesworth (I877). Ces trois contes témoignent, selon elle, d'une révolte contre le rôle assigné à la femme de cette époque, même si la forme littéraire reste ici assez conventionnelle. Karolyn Crowley et John Pennington se demandent, quant à eux, si la réécriture féministe des contes de fées ne constitue pas, au fond, une «fraude» à l'égard de l'esprit d'un genre, rétif au didactisme, comme l'avait souligné Dickens en son temps (Fraud on the Fairies, I853). Une étude du conte de Cendrillon selon ses reprises par Barbara Walker, Francesca Lia Block et Emma Donoghue suggère que les meilleurs contes féministes ne se réduisent jamais à leur message : "Sans repenser créativement le genre et la forme, une fraude féministe sur les fées semble inévitable.» (p. 3II)

La rubrique «Texts \& Translations» présente une curieuse adaptation de Rapunzel (Raiponce) par Marina Warner, sous le titre: The Difference in the Dose; on peut lire ensuite une dense réflexion de l'auteur sur le conte source et son propre travail d'écriture. On découvre ensuite trois belles variations graphiques de Rima Staines sur la figure de la vieille femme dans la forêt : Baba Yaga, la mère-grand du Chaperon rouge et la sorcière d'Hansel et Gretel. Elles sont accompagnées d'un texte de l'artiste présentant l'esprit de son travail. 